\title{
Periostin is Secreted by Glioblastoma CD90-positive Stromal Cells and Acts as a Pericyte Chemoattractant
}

Edona Emini ${ }^{1,2 \#}$, Tania Ramos-Moreno ${ }^{1,2 \#^{*}}$, Francesca Romana Stefani ${ }^{1,2}$, Andreas Svensson $^{1,2}$ and Johan Bengzon ${ }^{1,2,3}$

${ }^{1}$ Stem Cell Center, BMC B10, Lund University, Lund, Sweden

${ }^{2}$ Division of Neurosurgery, Department of Clinical Sciences, Lund University, Lund, Sweden

${ }^{3}$ Department of Neurosurgery, Skåne University Hospital, Lund, Sweden

\#Contributed equally to this work

\begin{abstract}
Glioblastoma (GBM) stroma is composed of multiple cell types including vascular elements, immune cells and mesenchymal stromal cells (MSCs). Periostin (POSTN) is a secreted extracellular matrix protein which plays a crucial role in the progression of this aggressive and highly vascularized tumor. However, the cellular distribution of gliomaderived POSTN and whether POSTN can act as a chemoattractant for tumor vasculogenic cells is not known. The aim of the present study was to identify the specific cellular distribution of POSTN within GBM and to explore the possibility of POSTN acting as an attractant for tumor pericytes. Here we show that POSTN expression by large is restricted to the stromal compartment of GL261 mouse GBM. Within the stroma, POSTN is mainly localized to CD90', most likely mesenchymal stromal cells (MSCs), and to pericytes recruited into the tumor. High POSTN protein levels were found to be produced by $\mathrm{CD}^{+} 0^{+}$MSCs acutely isolated from human GBM. Both mouse and human CD90+ MSCs co-expressed POSTN and Integrin $\beta 1$, permitting autocrine interaction between ligand and receptor. Pericytes expressing Integrin $\beta 1$ and $C D 90^{+}$perivascular cells expressing POSTN are adjacently localized within the mouse GL261 stroma. A large fraction of human brain pericytes were found to express Integrin $\beta 1$ and showed Integrin $\beta 1$ dependent migration in response to POSTN. In summary, our findings tie the expression and action of POSTN to the stromal compartment of GBM and support a role for POSTN in GBM angioproliferation.
\end{abstract}

Keywords: Glioblastoma multiforme; Periostin; Pericytes; Integrin $\beta 1$

\section{Introduction}

Glioblastoma multiforme (GBM) is the most common and aggressive brain tumor in adults. Despite intense research and clinical efforts, the median survival time for GBM patients undergoing conventional treatment (i.e., surgery, radiotherapy, and chemotherapy) is only 15 months [1]. This neoplasm is characterized by areas of hypoxia and necrosis and an extensive stromal component supporting hypoxia-induced neovascularization and tumor growth. The tumor stroma is composed of multiple cell types including vascular elements, immune cells and mesenchymal stromal cells (MSCs). Mesenchymal stromal cells have recently been isolated from GBM and characterized $[2,3]$. Less aggressive astroglial tumors in adults, i.e. astrocytoma grade II and anaplastic astrocytoma, do not contain necrotic areas and exhibit considerably less stroma and angioproliferation [4].

Periostin (POSTN) is a secreted extracellular matrix protein which plays a crucial role in GBM progression by promoting invasiveness [5] and angiogenesis [6] and plays a potential role in the clinical response to angiogenic therapy [7]. Intra-tumoral level of POSTN correlates with increasing glioma malignancy as well as an expanding stromal component. Importantly, POSTN levels have been shown to correlate to the risk of recurrence and inversely with patient overall survival [8]. Recently, it was shown that POSTN mediates the recruitment of tumorpromoting M2 macrophages in GBM [9-11]. However, the cellular sources for glioma-derived secreted POSTN and whether POSTN can also act as a chemoattractant for other cellular constituents of the GBM stroma is not known.

The aim of the present study is to identify the specific cellular distribution of POSTN within GBM and to explore the possibility that POSTN acts as an attractant for glioma vasculogenic pericytes.

\section{Material and Methods}

\section{Ethical statement}

All animal work was approved by the Committee of Animal Ethics in Lund-Malmö, Sweden (permit number: M259-12).

\section{Tumor inoculation}

Nine heterozygote rgs5 $5^{\mathrm{GFP} /+}$ female mice between $7-17$ weeks of age were inoculated with mouse glioma 261 (GL261) tumor model cells. The Rgs5 $5^{\mathrm{GFP} /+}$ mouse line is a knock-out/knock-in C57BL/6 mouse line where GFP is expressed under the pericyte-specific RGS5 promoter [12]. Prior to inoculation, mice were anaesthetized with isoflurane (Forene, Abbott, CA, USA) and positioned in a stereotaxic frame (David Kopf Instruments, Tujunga, CA, USA). Local anesthetic $(0.025 \mathrm{ml}$ of $2.5 \mathrm{mg} / \mathrm{ml}$ bupivacaine containing $5 \mu \mathrm{g} / \mathrm{ml}$ epinephrine (Marcain), AstraZeneca AB, Södertälje, Sweden) was subcutaneously injected on the head region, a $1 \mathrm{~cm}$ long sagittal skin incision was made

*Corresponding author: Tania Ramos Moreno, Stem Cell Center/Department of Clinical Sciences, Lund University, Klinikgatan 26, Lund, 221 84, Sweden Tel: +46-46-2223159; E-mail: tania.ramo_moreno@med.lu.se

Received February 12, 2018; Accepted February 24, 2018; Published February 28, 2018

Citation: Emini E, Ramos-Moreno T, Stefani FR, Svensson A, Bengzon J (2018) Periostin is Secreted by Glioblastoma CD90-positive Stromal Cells and Acts as a Pericyte Chemoattractanth. J Stem Cell Res Ther 8:414 doi: 10.4172/2157-7633.1000414

Copyright: $\odot 2018$ Emini E, et al. This is an open-access article distributed unde the terms of the Creative Commons Attribution License, which permits unrestricted use, distribution, and reproduction in any medium, provided the original author and source are credited. 
Citation: Emini E, Ramos-Moreno T, Stefani FR, Svensson A, Bengzon J (2018) Periostin is Secreted by Glioblastoma CD90-positive Stromal Cells and Acts as a Pericyte Chemoattractanth. J Stem Cell Res Ther 8: 414. doi: 10.4172/2157-7633.1000414

Page 2 of 6

and a hole was drilled in the skull. Five thousand GL261 tumor cells in 5 $\mu \mathrm{l} 0$ medium (RPMI 1640 medium supplemented with $1 \mathrm{mM}$ sodium pyruvate and $10 \mathrm{mM}$ HEPES) were injected using a $10 \mu \mathrm{l}$ syringe (Hamilton Bonaduz AG, Bonaduz, Switzerland) at $1 \mu \mathrm{l} / \mathrm{min}$ into the caudate nucleus. The following coordinates were used: $1.5 \mathrm{~mm}$ lateral and $1.0 \mathrm{~mm}$ anterior of bregma, $2.75 \mathrm{~mm}$ ventral of the skull bone. The needle was left in the brain for 5 minutes after the injection before it was slowly retracted and the hole in the skull was sealed with bone wax.

\section{Immunofluorescence}

At day 19 after tumor inoculation, animals were sacrificed by transcardial perfusion with $0.9 \% \mathrm{NaCl}$ solution (Merck $\mathrm{KGaA}$, Darmstadt, Germany) followed by $4 \%$ paraformaldehyde (PFA, Electron Microscopy Sciences, Hatfield, PA, USA). Brains were removed from the skull and postfixed in $4 \%$ PFA (Electron Microscopy Sciences, Hatfield, PA, USA) at $4^{\circ} \mathrm{C}$ overnight and thereafter transferred to $30 \%$ sucrose solution (Merck KGaA, Darmstadt, Germany). $40 \mu \mathrm{m}$ thick coronal sections were cut using a SM200 R sliding microtome (Leica Biosystems Nussloch GmbH, Nussloch, Germany). Coronal sections were stored at $-20^{\circ} \mathrm{C}$ in anti-freeze solution (30\% etylen glycol and $30 \%$ glycerol (both from VWR International, Radnor, PA, USA) in $0.012 \mathrm{M} \mathrm{NaH}_{2} \mathrm{PO}_{4} \cdot \mathrm{H}_{2} \mathrm{O}$ and $0.031 \mathrm{M} \mathrm{Na}_{2} \mathrm{HPO}_{4} \cdot 2 \mathrm{H}_{2} \mathrm{O}$ (both from Sigma-Aldrich, Stockholm, Sweden). After washing steps, free floating sections were first incubated in 10\% normal goat/donkey serum (NGS/NDS, Jackson ImmunoResearch Europe Ltd., Suffolk, United Kingdom Immuno Research) and 1\% Triton X-100 (Sigma Aldrich, Stockholm, Sweden) solution in potassium phosphate buffer saline (KPBS) buffer and then incubated over two nights at $4^{\circ} \mathrm{C}$ with rabbit anti-POSTN (1:400, Abcam, Cambridge, United Kingdom); mouse anti human-Integrin $\beta 1$ (1:400, Sigma Aldrich, Stockholm, Sweden); mouse anti-CD90 (1:200, Santa Cruz Biotechnology Inc, USA); chicken anti-GFP (1:400, Abcam, Cambridge, United Kingdom); mouse antiNestin (diluted 1:200, Merck Millipore, Billerica, MA, USA) in 3.3\% NGS (Sigma Aldrich, Stockholm, Sweden) and 0.3\% Triton $\mathrm{x}-100$ in KPBS. Sections were then washed in KPBS and incubated with suitable secondary antibodies in 3.3\% NGS/NDS and $0.3 \%$ Triton X-100 (Sigma Aldrich, Stockholm, Sweden) in KPBS for two hours in dark at room temperature. After a final washing step, sections were mounted on SuperFrost Plus glasses (Thermo Fisher Scientific Inc., Waltham, MA, USA) and covered with polyvinyl alcohol (PVA, Sigma Aldrich, Stockholm, Sweden)-1,4 diazabicyclo [2.2.2] octane (DABCO, Sigma Aldrich, Stockholm, Sweden) supplemented with Hoechst $33342(1 \mu \mathrm{l} /$ $\mathrm{ml}$, Sigma Aldrich, Stockholm, Sweden) for nuclear staining.

\section{Cell culture}

Isolation and culture of human MSC-like CD90 ${ }^{+}$and CD90cells from human glioma were recently reported by our group [3]. Briefly, cells were obtained from primary brain tumor surgery at the department of neurosurgery at Skane University Hospital in Lund, Sweden, Ethical permit H15 642/2008. Passage 2-4 primary cells, grown adherently on plastic, were sorted on a FACS Aria III cell sorter (BD Biosciences, Heidelberg, Germany) based on specific mesenchymal stromal cells defining markers (CD73, CD90, CD105 and HLA class I) with flow cytometry in a FACS Aria III cell sorter (BD Biosciences, Heidelberg, Germany). Doublets, dead cells and cells expressing lineage negative markers (CD14, CD19, CD34, CD45 and HLA-DR) were used as a cocktail in Lin TO-PRO-1 and excluded. These MSC markers are defined by The International Society for Cellular Therapy (ISCT; $[3,13])$. Cells were then expanded, frozen and kept at $-150^{\circ} \mathrm{C}$ until used.

For the present experiments, sorted $\mathrm{CD} 0^{+}$MSCs from passage
4-6 were cultured in MSC Expansion Media (StemCell Technologies, Cambridge, UK) supplemented with antibiotic-antimycotic solution (AAS, Sigma-Aldrich, Stockholm, Sweden) at $37^{\circ} \mathrm{C}$ and $5 \% \mathrm{CO}_{2}$.

Human brain vascular pericytes (HBVPs; $3 \mathrm{H}$ Biomedical, Uppsala, Sweden) were seeded in $2 \%$ gelatin-coated flasks and expanded in pericyte medium (3H Biomedical, Uppsala, Sweden).

\section{Immunocytochemistry (ICC)}

A total of $10^{5} \mathrm{CD}^{+} 0^{+}$and $10^{5} \mathrm{CD} 90^{-}$cells per well were cultured in 8 well chamber culture slides for $24 \mathrm{~h}$ and fixed with $4 \%$ PFA (Electron Microscopy Sciences, Hatfield, PA, USA) for $30 \mathrm{~min}$ at RT. After a subsequent blocking step using donkey serum (1:20 in DPBS (Life Technologies, Carlsbad, CA, USA) for $30 \mathrm{~min}$ at RT, cells were incubated with primary antibody for $2 \mathrm{~h}$ and $30 \mathrm{~min}$. Between each step, cells were washed with DPBS (Life Technologies, Carlsbad, CA, USA). Primary antibodies included mouse anti human-Nestin (1:400, Merck Millipore, Billerica, MA, USA); rabbit anti-POSTN (1:400, Abcam, Cambridge, United Kingdom); mouse anti-human Integrin $\beta 1$ (1:200, Sigma Aldrich, Stockholm, Sweden) and suitable secondary antibodies were then applied for $30 \mathrm{~min}$. After the staining, chambers were removed and sections were mounted using Gold Anti-Fade with DAPI (Thermo Fisher Scientific Inc., Waltham, MA, USA).

\section{Quantification and microscopical analysis}

For quantification of ICC ten randomized snapshots were taken in an Olympus BX61 (Olympus, Tokyo, Japan) epifluorescence microscope at $40 \mathrm{x}$ magnification. Snapshots were used for quantification using the software ImageJ (National Institutes of Health, Bethesda, Maryland). For immunoflourescence quantifications, the total number of $\mathrm{CD} 90^{+}$ MSCs, $\mathrm{POSTN}^{+}$and $\mathrm{GFP}^{+}$cells in the graft was determined by stereology using C.A.S.T.-Grid software in a total of 3 animals. For colocalization of CD90 and POSTN antibodies, around 500 cells per animal were counted in random fields from the graft area in an Olympus BX61 (Tokyo, Japan) epifluorescence microscope. Colocalization of different markers was previously validated using a Zeiss LSM 780 confocal microscope (Carl Zeiss Microscopy GmbH, Jena, Germany). Results are expressed as average of total numbers and/or percentage of total number \pm S.E.M.

\section{RNA sequencing}

Total RNA from $\mathrm{CD}^{+} 0^{+}$and $\mathrm{CD} 90^{-}$cells at passage number 5 and 8 , U87 primary GBM cell line (passage no. 30) and human bone-marrow derived MSCs (hBM-MSCs, passage no. 1, from a 61-year-old healthy male donor) was isolated using RNeasy (Qiagen) with DNase treatment according to manufacturer instructions. RNA concentration was determined by Nanodrop-ND 1000 spectrophotometer (Nanodrop) and RNA quality was analyzed with Bioanalyzer (Agilent). Samples with RNA integrity greater than seven were further amplified and analyzed as previously described in Ref. $[14,15]$ with exception of library preparation, for which we used TruSeq Stranded mRNA Kit for NeoPrep from Illumina.

\section{Enzyme-linked immunosorbent assay (ELISA)}

POSTN secretion from $\mathrm{CD}^{+} 0^{+}$(passage 8) and CD90- (passage 10) was measured by ELISA using the human POSTN ELISA kit (Thermo Fisher Scientific Inc., Waltham, MA, USA). Briefly, $10^{5}$ cells were seeded in each well in a 24 well plate and after $24 \mathrm{~h}$ incubation in MSC Expansion medium; supernatants were collected and immediately stored at $-80^{\circ}$ until use. ELISA was performed and analyzed according to the manufacturer's instructions. Absorbance was read at $450 \mathrm{~nm}$ and 
Citation: Emini E, Ramos-Moreno T, Stefani FR, Svensson A, Bengzon J (2018) Periostin is Secreted by Glioblastoma CD90-positive Stromal Cells and Acts as a Pericyte Chemoattractanth. J Stem Cell Res Ther 8: 414. doi: 10.4172/2157-7633.1000414

Page 3 of 6

550nm on SpectraMax M2 e microplate reader (Molecular Devices, LLC., Sunnyvale, CA, USA).

\section{Migration assay}

The migration assay was performed using a 24-well tissue culture plate where $8 \mu \mathrm{m}$ pore size (Corning, NY, USA) transwell inserts were placed into the wells. HBVPs $\left(10^{5}\right.$ cells/well) were then seeded into the insert and incubated for $18 \mathrm{~h}$ with recombinant POSTN (R\&D Systems, Inc., Minneapolis, USA) at the different concentrations of $50 \mathrm{ng}, 100$ $\mathrm{ng}$, and $200 \mathrm{ng} / \mathrm{ml}$. After this incubation step, non-migrating cells were gently removed and migrating cells that had passed through the filter were fixed with 4\% PFA (Electron Microscopy Sciences, Hatfield, PA, USA), permeabilized with $1 \%$ TX-100 and stained with Hoechst 33342. Next, the filter was cut out and mounted on a microscope slide for counting nuclei under an Olympus BX61 epifluorescence microscope (Tokyo, Japan). Ten randomly distributed spots were snapshot using 20x magnification and nuclei were counted using the Image J software (National Institutes of Health, Bethesda, Maryland).

For the Integrin $\beta 1$ blocking experiment, cells were incubated with monoclonal mouse anti-human Integrin $\beta 1$ antibody (1:200, Sigma
Aldrich, Stockholm, Sweden) for $3 \mathrm{~h}$ at $37^{\circ} \mathrm{C}$ prior to the transwell migration assay, where a POSTN concentration of $200 \mathrm{ng} / \mathrm{ml}$ was used. As control, the cells were incubated with pericyte medium without blocking antibody.

\section{Statistical analysis}

For RNA sequencing data processing the analysis pipe line method described in SCAN-B article was used as previously described in Ref. [3]. Student's t-test was used for between group analysis of ELISAdata and cell numbers $p<0.05$ was considered significant. Results are expressed as mean \pm S.E.M.

\section{Results}

\section{POSTN expression in GBM tumor stroma}

First, we investigated the expression of POSTN in GBM tumor stroma and asked if POSTN colocalizes with $\mathrm{CD} 90^{+}$cells by means of immunohistochemistry in brain tissue collected from $\mathrm{rgs}^{\mathrm{GFP} /+}$ that received GL261 cell inoculation. Since GFP is expressed under the pericyte-specific RGS5 promoter the $\operatorname{rgs}^{\mathrm{GFP} / \mathrm{+}}$ [12] we can distinguish what is the fraction of pericytes that express CD90 and what can belong to the stroma (based on [16]). Stereological quantification showed that $68.3 \pm 2.2 \%$ of the cells positive for POSTN co-express CD90 and 21.6

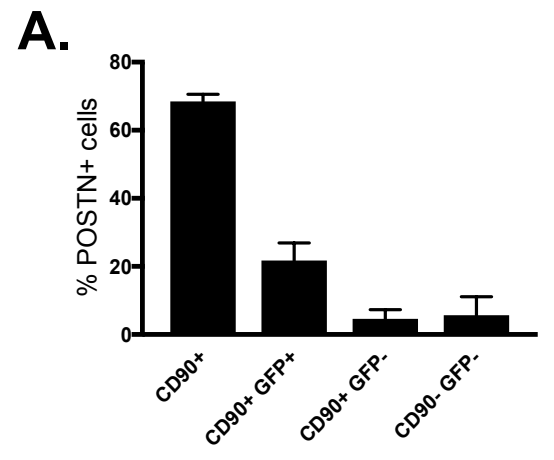

B.
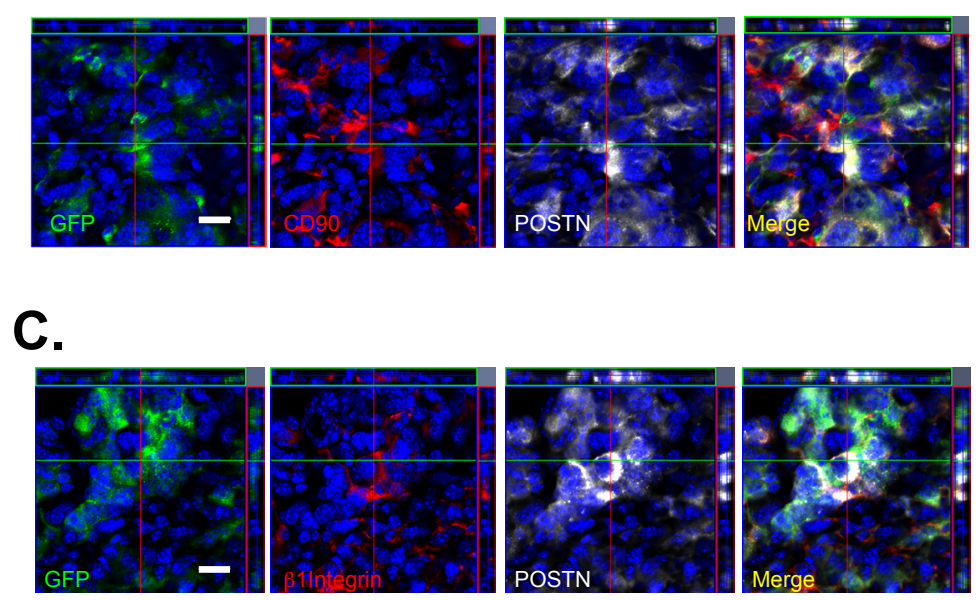

Figure 1. POSTN expression in GBM after inoculation of GL261 in rgs ${ }^{5 \mathrm{GFP} /+}$ animal model. A. Stereological quantification shows that $68.3 \pm 2.2 \%$ of the cells positive for POSTN coexpress CD90. Moreover, $21.6 \pm 5.2 \%$ are GFP ${ }^{+} \mathrm{CD} 0^{+}$cells; $4.5 \pm 2.8 \%$ are GFP ${ }^{+} \mathrm{CD}^{-} 0^{-}$cells and $5.5 \pm 5.5 \%$ are cells that are GFP-CD90- $(n=3$ animals). B. Representative confocal image showing the intratumoral localization of POSTN in CD90 MSCs. Scale bar: 20 um. C. Representative confocal image showing the relation of POSTN and integrin $\beta 1$ within the tumor. Note that Integrin $\beta 1$ is, in addition, expressed in GFP ${ }^{+}$pericytes. Scale bar: $20 \mu \mathrm{m}$. 
Citation: Emini E, Ramos-Moreno T, Stefani FR, Svensson A, Bengzon J (2018) Periostin is Secreted by Glioblastoma CD90-positive Stromal Cells and Acts as a Pericyte Chemoattractanth. J Stem Cell Res Ther 8: 414. doi: 10.4172/2157-7633.1000414

Page 4 of 6

$\pm 5.2 \%$ of these $\mathrm{CD} 90^{+}$cells express GFP as well, indicating a pericytic phenotype. A minority of the $\mathrm{POSTN}^{+}$cells are $\mathrm{GFP}^{+} \mathrm{CD}^{-}$cells $(4.5 \pm$ $2.8 \%$ ) whilst $5.5 \pm 5.5 \%$ are GFP CD90 cells (Figure $1 \mathrm{~A}$ and $1 \mathrm{~B}$ ).

Since $21.6 \pm 5.2 \%$ of the $\mathrm{CD} 90^{+}$cells were pericytes, we next asked if the Integrin $\beta 1$, a receptor for POSTN expressed by pericytes $[17,18]$, associates with POSTN in vivo. As shown in Figure $1 \mathrm{~A}$ and $1 \mathrm{C}$, Integrin $\beta 1$ is expressed on $\mathrm{CD}^{+} 0^{+}$pericytes and closely associates with $\mathrm{POSTN}^{+}$ cells (Figure 1C).

In summary, both POSTN and its receptor Integrin $\beta 1$ are mainly localized to $\mathrm{CD} 90^{+}$perivascular cells within mouse GL261 GBM.

\section{Periostin is secreted in the tumor stroma by $\mathrm{CD}^{+} 0^{+}$stromal} cells

Based on immunofluorescence analysis and on our previous finding of the presence of two GBM stromal MSC clones differing in their CD90 expression [3], we next asked if $\mathrm{CD} 90^{+}$MSCs could be a major source for POSTN secretion. To address this question, we performed RNAseq using the $\mathrm{CD} 90^{+}$and $\mathrm{CD} 90^{-}$MSCs derived from 2 different human GBM samples (referred to as $47 \mathrm{p}$ and $48 \mathrm{p}$ and described), and compared their mRNA levels for POSTN between them and to the bulk brain tumor cells, U87 human brain tumor cell line and human bone marrow stromal cells (please see Ref. [3] for a more complete
A.

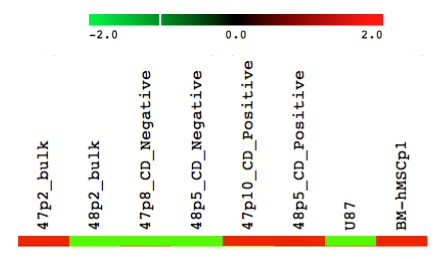

B.

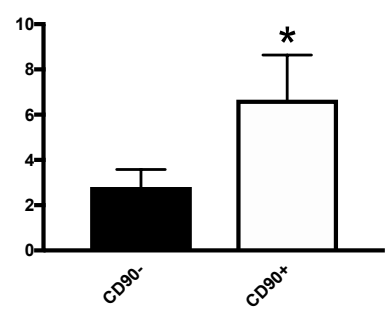

E.

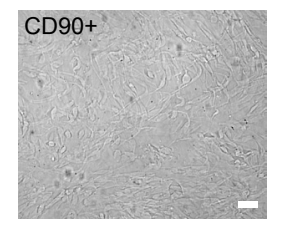

C.

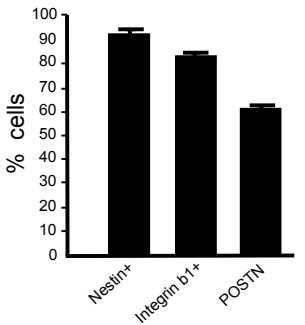

F.

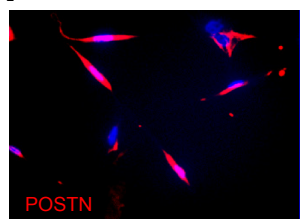

D.
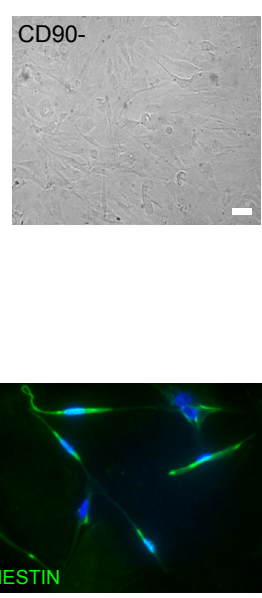

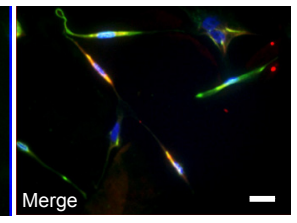

G.
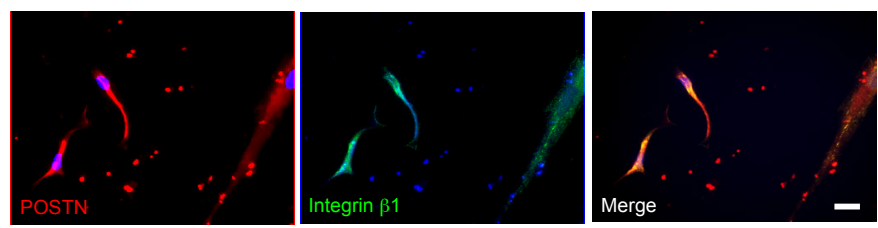

Figure 2: POSTN expression in acutely isolated cultured human MSCs from glioblastoma patients. A. Heatmap for POSTN gene showing the differential expression in RNA-sequencing analysis performed on two patients' primary tumor samples, named GBM-47 and GBM-48; GBM-47/48-derived MSC-like CD90- cells; GBM47/48-derived MSC-like CD90+ cells; U87 human primary GBM cell line and bone marrow derived MSCs. Note that, although not enough number of patients to make the difference to be significantly, glioma derived MSC-like CD90+ cells' POSTN expression is higher than their counterparts GBM-47/48-derived MSC-like CD90- cells ( $p>0.01$; Student's $t$ test). B. ELISA analysis supports the RNAseq results for POSTN and confirms that POSTN secretion levels are higher in human CD90 ${ }^{+}$MSCs (CD90- MSCs: $2.8 \pm 1.6$ vs CD90 MSCs $6.6 \pm 1.8 ; p<0.05, n=3$ independent experiments, 5 technical replicates). C. POSTN, Nestin and Integrin $\beta 1$ expression in CD90 MSCs in vitro shows that $94 \pm 1.9 \%$ of the cells are positive for Nestin; $84 \pm 3.1 \%$ are positive for Integrin $\beta 1$ and $62 \pm 6.7 \%$ are positive for POSTN ( $\mathrm{n}=1$ independent experiment, 3 technical replicates). D. Representative picture of CD90-MSCs in bright field. Scale bar: $50 \mu \mathrm{m}$. E. Representative picture of CD90+ MSCs in bright field. Scale bar: $50 \mu \mathrm{m}$. F. Representative picture of Nestin and POSTN expression in CD90 ${ }^{+}$MSCs. Scale bar: $20 \mu \mathrm{m}$.

G. Representative picture of POSTN and Integrin $\beta 1$ expression in CD90+MSCs. Scale bar: $20 \mu \mathrm{m}$. 
characterization of the $\mathrm{CD}^{+} 0^{+}$and $\left.\mathrm{CD} 90^{-} \mathrm{MSCs}\right)$. mRNAseq analysis confirmed that the expression of POSTN is higher in GBM CD90 MSCs compared to CD90- MSCs (Figure 2A).

We then investigated whether $\mathrm{CD} 90^{+}$MSCs secrete POSTN. ELISA analysis on cell culture supernatant from $\mathrm{CD}^{+} 0^{+}$and $\mathrm{CD} 90^{-} \mathrm{MSC}$ showed that POSTN secretion is significantly higher in $\mathrm{CD}^{\circ} 0^{+} \mathrm{MSCs}$ (6.6 \pm 1.8$)$ compared to CD90- MSCs $(2.8 \pm 1.6$; Figure 2B, $\mathrm{p}<0.05)$

Finally, CD90 ${ }^{+}$MSCs were were subsequently subjected to immunocytochemical studies. Quantitative analysis revealed that $94 \pm$ $1.9 \%$ of the cells expressed Nestin, a stem cell and MSC marker whilst $84 \pm 3.1 \%$ expressed Integrin $\beta 1$ and $62 \pm 6.7 \%$ cells expressed POSTN (Figure $2 \mathrm{C}-2 \mathrm{G}$ ). In brief, $\mathrm{CD} 90^{+} \mathrm{MSC}$ acutely isolated from human GBM secrete POSTN.

\section{POSTN acts as a chemoattractant for pericytes}

Our present results showing that $\mathrm{GFP}^{+}$pericytes express Integrin $\beta 1$ and this membrane receptor colocalizes with POSTN (present results) lead us to hypothesize that POSTN might act as a chemoattractant for human brain pericytes. For this experiment, we used a concentration series of recombinant human POSTN of $50 \mathrm{ng}, 100 \mathrm{ng}$, and $200 \mathrm{ng} / \mathrm{ml}$ based on previous work [15]. Results show that pericyte migration is stimulated at a concentration of $200 \mathrm{ng} / \mathrm{ml}$ POSTN (Figure 3A, $\mathrm{p}<0.05$ ). To confirm that human brain pericyte migration is mediated by POSTN via Integrin $\beta 1$ we looked if the migration would be reduced by blocking Integrin $\beta 1$. For this purpose, pericytes were incubated for $3 \mathrm{~h}$ with an Integrin $\beta 1$ antibody prior to the migration assay. As shown in Figure $3 \mathrm{~B}$, the migration was significantly reduced after blocking the receptor, indicating that Integrin $\beta 1$ is involved in the POSTN-induced pericyte migration $(\mathrm{p}<0.05)$. In summary, POSTN may act as a chemoattractant for human brain pericytes via Integrin $\beta 1$.

\section{Discussion}

The present study demonstrates that POSTN expression is largely restricted to the perivascular stromal niche of GL261 mouse glioblastoma. Within this compartment, POSTN expression is predominantly localized to $\mathrm{CD} 90^{+}$putative MSCs and to pericytes recruited into the tumor. This stromal expression goes in line with previous work in pancreatic cancer showing that stromal cells are the source of POSTN [19]. The findings in the mouse glioma model are further confirmed in human tissue, specifically by CD $90^{+}$MSCs acutely isolated from glioblastoma being a source of POSTN.

Present data also show that both mouse and human $\mathrm{CD} 90^{+}$MSCs coexpress POSTN and Integrin $\beta 1$, permitting autocrine interaction between ligand and receptor. Pericytes expressing Integrin $\beta 1$ closely associate with $\mathrm{CD} 90^{+}$perivascular cells expressing POSTN within the mouse GL261 stroma. Similarly, a large fraction of human brain pericytes express Integrin $\beta 1$, showing Integrin $\beta 1$-dependent migration in response to POSTN.

POSTN is now recognized as an important component of the tumor stroma and a biomarker and prognostic determinator in several types of cancer including GBM (reviewed in Ref. [20]). In human glioma, POSTN expression levels have previously been correlated directly with tumor grade and recurrence, and inversely with survival [8]. POSTN plays a pivotal role in tumor growth as its knockdown markedly impairs glioblastoma growth in a mouse xenograft model [8]. The present work defines the cellular distribution of this important protein to the CD90 ${ }^{+}$ stromal niche within the GBM microenvironment. Supporting our finding of POSTN expression within the angioproliferative stroma of GBM, the work by Mikheev, et al. [8] demonstrated that, although low-level POSTN expression was detected in low grade glioma, the expression was several log-folds higher in high grade (grade IV) compared to low grade glioma (grade II). Furthermore, stromal
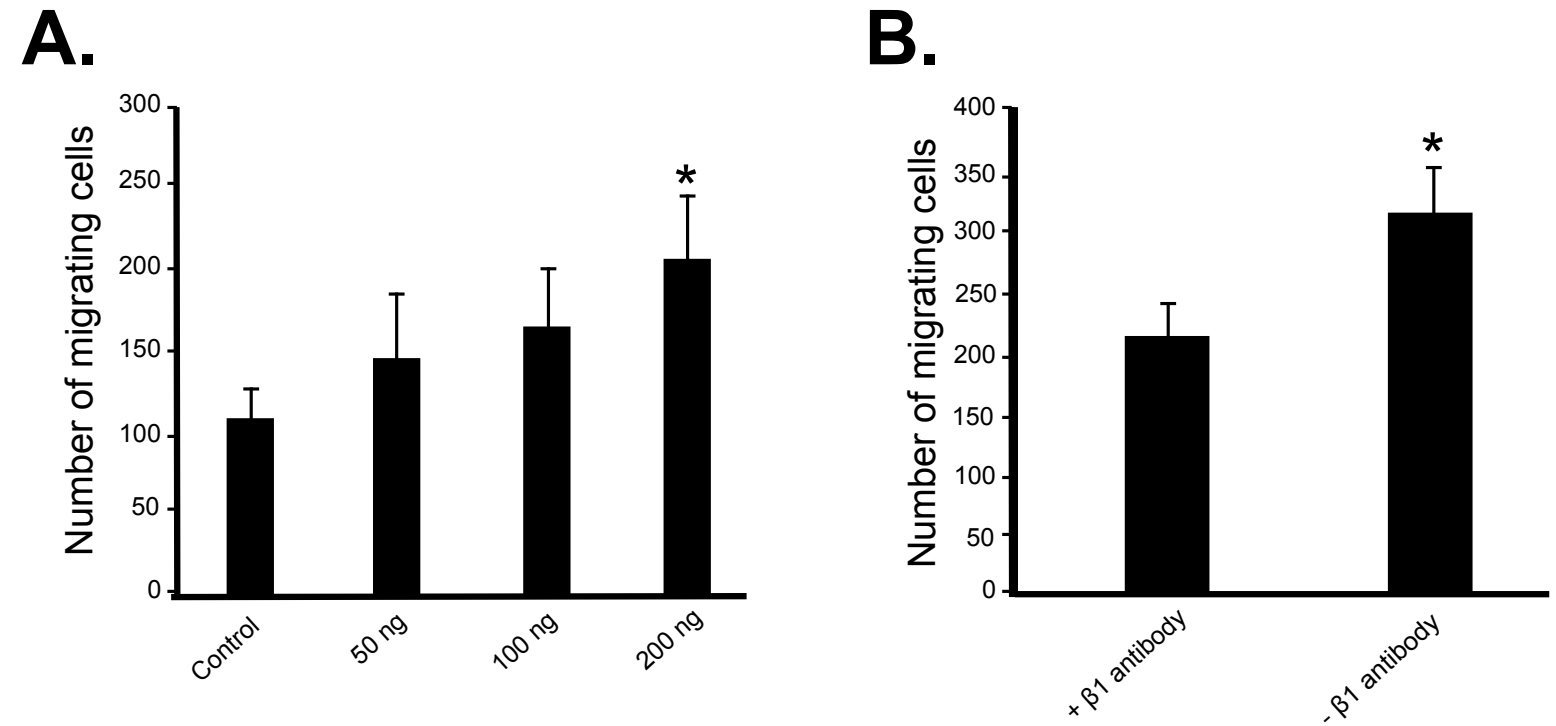

Figure 3. POSTN acts as a chemoattractant for pericytes via Integrin $\beta 1$. A. Transwell migration assay shows the highest migration of pericytes at a POSTN concentration of $200 \mathrm{ng} / \mathrm{ml}$ (mean of migrated cells in the control experiment: $108.7 \pm 17$; concentration of $50 \mathrm{ng} / \mathrm{ml}$ : $144.9 \pm 39.6$; concentration of $100 \mathrm{ng} / \mathrm{ml}$ : 163.7 \pm 33.2 ; concentration of $200 \mathrm{ng} / \mathrm{ml} ; 205.11 \pm 42$. $n=3$ experimental replicates, in technical duplicates; $p<0.05)$. B. Transwell migration assay after blocking Integrin $\beta 1$ shows significantly decreased migration of pericytes (mean of migrated cells: (-)Integrin $\beta 1$ antibody: $309 \pm 44 ;(+)$ Integrin $\beta 1$ : $212 \pm 35$. $n=3$ experimental replicates, in technical duplicates; $p<0.05$ ) 
Citation: Emini E, Ramos-Moreno T, Stefani FR, Svensson A, Bengzon J (2018) Periostin is Secreted by Glioblastoma CD90-positive Stromal Cells and Acts as a Pericyte Chemoattractanth. J Stem Cell Res Ther 8: 414. doi: 10.4172/2157-7633.1000414

Page 6 of 6

deposition of POSTN was detected only in GBM, with the highest levels found in gliosarcoma, the most aggressive type of glioma, displaying distinct mesenchymal features.

POSTN released by GBM cells under hypoxic conditions has been shown to recruit M2 macrophages into the tumor microenvironment and in this way promote tumor growth [9]. Interestingly, tumorassociated macrophages stimulate microvessel proliferation in glioma by activation of pericytes [21]. The present study focuses on the role of POSTN, and its receptor Integrin $\beta 1$, in the recruitment of tumorassociated pericytes. In previous work we have demonstrated that, in a mouse GBM model, pericytes are recruited from widespread areas of the brain into glioblastoma and constitute a major part of the tumor pericyte population [3]. In humans, hyperplasia of pericytes is one of the significant characteristics of malignant glioma [22]. In the present work, we found that human brain pericytes migrate by Integrin $\beta 1$ dependent POSTN signaling, opening up to the possibility that tumor pericytes are recruited in vivo through this mechanism. Moreover, and adding to our previous work where we showed that human CD90 stromal cells secrete the potent vasculogenic molecule VEGF that call epithelial cells (EC) to form new tubes $[3,23,24]$, present results shows a parallel and distinctive role for the $\mathrm{CD} 90^{+}$mesenchymal stromal cells which, by means of secreting POSTN, would be attracting pericytes to the endothelial newly formed tubes. This pericyte recruitment would induce capillary maturation by inducing the formation of a new basal lamina [25-27].

In summary, our findings tie the expression and action of POSTN to the mesenchymal component of GBM and suggest an important role for POSTN in glioblastoma stromal cell support of tumor angiogenesis via Integrin $\beta 1$-dependent POSTN signaling.

\section{Acknowledgements}

This work was supported by donations by Viveca Jeppsson and MajBritt and Allan Johansson, ALF grants and Region Skåne.

\section{References}

1. Stupp R (2002) Promising survival for patients with newly diagnosed glioblastoma multiforme treated with concomitant radiation plus temozolomide followed by adjuvant temozolomide. J Clin Oncol 20: 1375-82. [PubMed]

2. Hossain A (2015) Mesenchymal Stem Cells Isolated From Human Gliomas Increase Proliferation and Maintain Stemness of Glioma Stem Cells Through the IL-6/gp130/STAT3 Pathway. Stem Cells 33: 2400-2415. [PubMed]

3. Svensson A (2017) Identification of two distinct mesenchymal stromal cell populations in human malignant glioma. J Neurooncol 131: 245-254. [PubMed]

4. Louis DN (2007) The $2007 \mathrm{WHO}$ classification of tumours of the central nervous system. Acta Neuropathol 114: 97-109. [Pub Med]

5. Wang HY, Wang C, Jiang C (2013) Stromal protein periostin identified as a progression associated and prognostic biomarker in glioma via inducing an invasive and proliferative phenotype. Int J Oncol 42: 1716-1724. [PubMed]

6. Mustafa DA (2012) A proteome comparison between physiological angiogenesis and angiogenesis in glioblastoma. Mol Cell Proteomics 11: M111.008466. [PubMed]

7. Park SY (2016) Periostin (POSTN) Regulates Tumor Resistance to Antiangiogenic Therapy in Glioma Models. Mol Cancer Ther 15: 2187-2197. [PubMed]
8. Mikheev AM (2015) Periostin is a novel therapeutic target that predicts and regulates glioma malignancy. Neuro Oncol 17: 372-382. [PubMed]

9. Guo X (2016) Hypoxia promotes glioma-associated macrophage infiltration via periostin and subsequent M2 polarization by upregulating TGF-beta and M-CSFR. Oncotarge 7: 80521-80542. [PubMed]

10. Shi $Y$ (2015) Hostile takeover: glioma stem cells recruit TAMs to support tumor progression. Cell Stem Cell 16: 219-220. [PubMed]

11. Zhou W (2015) Periostin Secreted by Glioblastoma Stem Cells Recruits M2 Tumor-associated Macrophages and Promotes Malignant Growth. Nature cell biology, 2015. 17: 170-182. [PubMed]

12. Nisancioglu $\mathrm{MH}$ (2008) Generation and characterization of rgs 5 mutant mice Mol Cell Biol 28: 2324-2331. [PubMed]

13. Dominici M (2006) Minimal criteria for defining multipotent mesenchyma stromal cells. The International Society for Cellular Therapy position statement. Cytotherapy, 2006 8: 315-317. [PubMed]

14. Saal LH (2015) The Sweden Cancerome Analysis Network - Breast (SCAN-B) Initiative: a large-scale multicenter infrastructure towards implementation of breast cancer genomic analyses in the clinical routine. Genome Med 7: 20. [PubMed]

15. Hakkinen J (2016) Implementation of an Open Source Software solution for Laboratory Information Management and automated RNAseq data analysis in a large-scale Cancer Genomics initiative using BASE with extension package Reggie. bioRxiv: 038976.

16. Park TI (2016) Cultured pericytes from human brain show phenotypic and functional differences associated with differential CD90 expression. Sci Rep 6: 26587. [PubMed]

17. Utispan K (2012) Periostin activates integrin alpha5beta1 through a PI3K/ AKTdependent pathway in invasion of cholangiocarcinoma. Int J Oncol 41 : 1110-1118. [PubMed]

18. Abraham S (2008) Integrin beta1 subunit controls mural cell adhesion, spreading, and blood vessel wall stability. Circ Res 102: 562-570. [PubMed]

19. Kanno A (2008) Periostin, secreted from stromal cells, has biphasic effect on cell migration and correlates with the epithelial to mesenchymal transition of human pancreatic cancer cells. Int J Cancer 122: 2707-2718. [PubMed]

20. Moniuszko T (2016) Role of periostin in esophageal, gastric and colon cancer Oncol Lett 12: 783-787. [PubMed]

21. Zhu C (2017) CECR1-mediated cross talk between macrophages and vascular mural cells promotes neovascularization in malignant glioma. Oncogene 36:5356-5368. [PubMed]

22. Sun $\mathrm{H}$ (2014) Hyperplasia of pericytes is one of the main characteristics of microvascular architecture in malignant glioma. PLoS One 9: e114246. [PubMed]

23. Krock BL, Skuli N, Simon MC (2011) Hypoxia-induced angiogenesis: good and evil. Genes Cancer 2: 1117-1133. [PubMed]

24. Srinivasan S (2015) Hypoxia-induced expression of phosducin-like 3 regulates expression of VEGFR-2 and promotes angiogenesis. Angiogenesis 18: 449462. [PubMed]

25. Stratman AN (2009) Pericyte recruitment during vasculogenic tube assembly stimulates endothelial basement membrane matrix formation. Blood 114: 5091 5101. [PubMed]

26. Stratman AN (2010) Endothelial-derived PDGF-BB and HB-EGF coordinately regulate pericyte recruitment during vasculogenic tube assembly and stabilization. Blood 116: 4720-4730. [PubMed]

27. Armulik A, Genove G, Betsholtz C (2011) Pericytes: developmental physiological, and pathological perspectives, problems, and promises. Dev Cell 21: 193-215. [PubMed] 